\title{
APPLICATION OF HM-NETWORKS WITH POSITIVE AND NEGATIVE HETEROGENEOUS REQUESTS FOR FINDING MEMORY VOLUME IN INFORMATION SYSTEMS
}

\author{
Mikhail Matalytski ${ }^{1}$, Pawel Zajac ${ }^{1}$, Dmitry Kopats ${ }^{2}$ \\ ${ }^{1}$ Institute of Mathematics, Czestochowa University of Technology \\ Czestochowa, Poland \\ ${ }^{2}$ Faculty of Mathematics and Computer Science, Grodno State Univercity \\ Grodno, Belarus \\ m.matalytski@gmail.com,pawel_zajac@vp.pl,dk80395@mail.ru
}

Received: 26 February 2019; Accepted: 12 April 2020

\begin{abstract}
To determine the memory volume of information systems (IS), it was proposed to use a stochastic model, based on the use of a queueing network with bypasses, known as an HM-network. This model includes the servicing of heterogeneous requests along with their volumes, the ability to change the volume of such requests with their passage of time and the possibility of finding computer viruses in them, and therefore servicing requests can be interrupted at some random time. The expressions were obtained for the expected total values of the volume of requests in IS nodes.
\end{abstract}

MSC 2010: 90B22, 60K25, 68M20

Keywords: HM-network, information systems, volumes of requests, positive and negative heterogeneous request

\section{Introduction}

In IS, the total volume of memory is limited to a certain value, which is usually called memory capacity [1]. When designing systems, the main task is to find the expected volume of memory to take into account the conditions that limit the share of lost information. One of the methods for solving problems of IS design is the use of HM-queueing networks (QN). According to IS, we will continue to understand systems as information transformation objects, delivered in the form of messages (requests) [1]. HM-networks can be used to determine the volume of a buffer storage of systems that are representing processing IS nodes and transferring demands.

Neglect time dependence of requests processing of their volumes can lead to serious errors in finding the buffer memory in IS. The solution in the general case of the above problems can be based on the use of HM-networks with requests of many types (heterogeneous requests) and bypasses. In such networks, the request 
during the transition from one queueing system (QS) to another brings some bypass last (which is equal to the volume of this request), and the bypass (volume) of the first QS is reduced by this amount.

Note that the method of finding non-stationary probabilities and average characteristics of G-networks, i.e. networks with positive and negative requests, operating under conditions of high load using the apparatus of multidimensional generating functions described in monograph [2]. The HM-network of such type was also investigated (in this case networks with positive and negative only homogeneous requests were considered). For the first time, the use of HM-networks to estimate memory in IS has been described in [3]. In [4], the method of finding the expected volume of homogeneous requests in open HM networks with a limited number of waiting places and bypasses of requests of the servicing systems was described, in [5] - HM-networks with the limited sojourn time of the requests in the queues, and in [6] - HM-networks with unreliable service of requests in systems.

Open networks with two classes of requests - "positive" and "negative" - were proposed in [7] (they are now called G-networks). In [8,9], G-network analysis with positive and negative heterogeneous reports in a stationary mode was carried out.

Consider an open network with $n$-one-line systems to which $r$-type requests come. To the $S_{i}$ system from the outside (from the $S_{0}$ system), the simplest stream of positive $c$-type requests with intensity $\lambda_{0 i c}^{+}$and the simplest stream of negative $c$-type requests with intensity $\lambda_{0 i c}^{-}$will enter, $i=\overline{1, n}, c=\overline{1, r}$. All stream requests in the network are independent. Requests come to the service randomly, i.e. if there are $s$-type $k_{i s}$-requests in the $i$-th QS, then the probability that the $c$-type request will be serviced is equal to $\frac{k_{i c}}{r}, i=\overline{1, n}, c=\overline{1, r}$. The times of servicing positive $\sum_{s=1}^{r} k_{i s}$

$c$-type requests that join in QS $S_{i}$ are exponential distribution with parameter $\mu_{i c}$, $i=\overline{1, n}, c=\overline{1, r}$. Negative $c$-type request, which enters the network system, in which there is even one positive $c$-type request, will destroy one of them of the same type.

Each positive $c$-type request enters QS $S_{i}$ with a probability $p_{0 i c}^{+}$, and negative with a probability $p_{0 i c}^{-}, \sum_{i=1}^{n} \sum_{c+!}^{r} p_{0 i c}^{+}=\sum_{i=1}^{n} \sum_{c+!}^{r} p_{0 i c}^{-}=1, i=\overline{1, n}, c=\overline{1, r}$. The positive $c$-type request after servicing in QS $S_{i}$ with the probability $p_{i c j s}^{+}$passes into QS $S_{j}$ as the positive $s$-type, with the probability $p_{i c j s}^{-}$- as the negative $s$-type request and with probability $p_{i c 0}=1-\sum_{j=1}^{n} \sum_{s+!}^{r}\left(p_{i c j s}^{+}+p_{i c j s}^{-}\right)$leaves the network and goes outside (in QS $S_{0}$ ) $i, j=\overline{1, n}, \quad s, c=\overline{1, r}$. The network status is described by the $\vec{k}=\left(k_{11}, k_{12}, \ldots, k_{1 r}, k_{21}, k_{22}, \ldots, k_{2 r}, k_{n 1}, k_{n 2}, \ldots, k_{n r}\right)$ vector $n r$ dimension, where $k_{i c}$ - the number of requests in QS $S_{i} c$-type, $i=\overline{1, n}, c=\overline{1, r}$. 


\section{Analysis of HM-Markov networks with positive and negative requests of different classes}

Consider now our network, taking into account changes in the total volume of requests in systems when servicing positive and negative requests. Consider the case when changes in the volume of requests related to transitions between network states are deterministic functions, depending on the network and time states. Let $I_{i c}$ be the dimension vector $n r$ with zero coordinates except the coordinate with the number $r(i-1)+c$, which equals $1 ; v_{i}(\vec{k}, t)$ - total volume of requests in the $i$-th QS system at time $t$ if the network was in the $\vec{k}$-state at the time of the initial; $u(x)$ - Heaviside step function, $\lambda^{+}=\sum_{i=1}^{n} \sum_{c=1}^{r} \lambda_{0 i c}^{+}, \lambda^{-}=\sum_{i=1}^{n} \sum_{c=1}^{r} \lambda_{0 i c}^{-}$. Possible transitions between network states, probability of transitions and changes of the total volume of network system requests related to these transitions are recorded in Table 1.

Table 1. Possible transitions between network states, probability of transitions and changes of the total volume of requests in the system $S_{i}$

\begin{tabular}{|c|c|c|}
\hline $\begin{array}{c}\text { Possible transitions } \\
\text { between network states }\end{array}$ & Probability of transitions & $\begin{array}{l}\text { Changes of the total } \\
\text { volume of requests in the } \\
\text { system } S_{i} \text { related to } \\
\text { transitions between } \\
\text { network states }\end{array}$ \\
\hline$(k, t) \rightarrow(k, t+\Delta t)$ & $1-\left[\lambda^{+}+\lambda^{-}+\sum_{i=1}^{n} \sum_{c=1}^{r} \mu_{i c} u\left(k_{i c}(t)\right)\right] \Delta t+o(\Delta t)$ & $r_{i}(k) \Delta t+v_{i}(k, t)$ \\
\hline $\begin{array}{c}(k, t) \rightarrow\left(k+I_{j s}, t+\Delta t\right), \\
j \neq i, s \neq c\end{array}$ & $\lambda_{0 j s}^{+} \Delta t+o(\Delta t)$ & $r_{i}(k) \Delta t+v_{i}\left(k+I_{j s}, t\right)$ \\
\hline $\begin{array}{c}(k, t) \rightarrow\left(k-I_{j s}, t+\Delta t\right) \\
j \neq i\end{array}$ & $\begin{array}{l}\mu_{j s} \frac{k_{j s}(t)+1}{\sum_{s^{*}=1}^{r} k_{j s}{ }^{*}(t)+1} p_{j s 0} u\left(k_{j s}(t)\right) \Delta t+ \\
+\lambda_{0 j s}^{-} u\left(k_{j s}(t)\right) \Delta t+\mu_{j s} \frac{k_{j s}(t)+1}{\sum_{s^{*}=1}^{r} k_{j s}(t)+1} \times \\
\times \sum_{\substack{m=1 \\
m \neq i}}^{n} p_{j s m c}^{-}\left(1-u\left(k_{m c}(t)\right)\right) \Delta t+o(\Delta t)\end{array}$ & $r_{i}(k) \Delta t+v_{i}\left(k-I_{j s}, t\right)$ \\
\hline$(k, t) \rightarrow\left(k+I_{i c}, t+\Delta t\right)$ & $\lambda_{0 i c}^{+} \Delta t+o(\Delta t)$ & $r_{0 i c}\left(k+I_{i c}, t\right)+v_{i}\left(k+I_{i c}, t\right)$ \\
\hline
\end{tabular}


Cont. Table 1

\begin{tabular}{|c|c|c|}
\hline$(k, t) \rightarrow\left(k-I_{i c}, t+\Delta t\right)$ & $\begin{array}{c}\mu_{i c} \frac{k_{i c}(t)+1}{\sum_{\substack{* \\
c^{*}=1}}^{r} k_{i c}(t)+1} p_{i c 0} u\left(k_{i c}(t)\right) \Delta t+ \\
+\lambda_{0 i c}^{-} u\left(k_{i c}(t)\right) \Delta t+\mu_{i c} \frac{k_{i c}(t)+1}{\sum_{\substack{* \\
c^{*}=1}}^{r} k_{i c}{ }^{*}(t)+1} \times \\
\times \sum_{\substack{c=1 \\
c \neq i}}^{n} p_{i c}^{-}\left(1-u\left(k_{c}(t)\right)\right) \Delta t+o(\Delta t)\end{array}$ & $\begin{array}{c}-R_{i c 0}\left(k-I_{i c}, t\right)+ \\
\quad+v_{i}\left(k-I_{i c}, t\right)\end{array}$ \\
\hline $\begin{array}{c}(k, t) \rightarrow \\
\left(k+I_{i c}-I_{j s}, t+\Delta t\right) \\
j \neq i, s \neq c\end{array}$ & $\mu_{j s} \frac{k_{j s}(t)+1}{\sum_{s^{*}=1}^{r} k_{j s}{ }^{*}(t)+1} p_{j s i c}^{+} u\left(k_{j s}(t)\right) \Delta t+o(\Delta t)$ & $\begin{array}{l}r_{i c j s}\left(k+I_{i c}-I_{j s}, t\right)+ \\
+v_{i}\left(k+I_{i c}-I_{j s}, t\right)\end{array}$ \\
\hline $\begin{array}{c}(k, t) \rightarrow \\
\left(k-I_{i c}+I_{j s}, t+\Delta t\right) \\
j \neq i, s \neq c\end{array}$ & $\mu_{i c} \frac{k_{i c}(t)+1}{\sum_{c^{*}=1}^{r} k_{i c}{ }^{*}(t)+1} p_{i c j s}^{+} u\left(k_{i c}(t)\right) \Delta t+o(\Delta t)$ & $\begin{array}{l}-r_{j s i c}\left(k-I_{i c}+I_{j s}, t\right)+ \\
\quad+v_{i}\left(k-I_{i c}+I_{j s}, t\right)\end{array}$ \\
\hline $\begin{array}{c}(k, t) \rightarrow \\
\left(k+I_{m l}-I_{d h}, t+\Delta t\right) \\
m, d \neq i, l, h \neq c\end{array}$ & $\mu_{m l} \frac{k_{m l}(t)+1}{\sum_{l^{*}=1}^{r} k_{m l^{*}}(t)+1} p_{m l d h}^{+} u\left(k_{d h}(t)\right) \Delta t+o(\Delta t)$ & $\begin{array}{c}r_{i}(k) \Delta t+ \\
+v_{i}\left(k+I_{m l}-I_{d h}, t\right)\end{array}$ \\
\hline $\begin{array}{c}(k, t) \rightarrow \\
\left(k-I_{i c}-I_{j s}, t+\Delta t\right) \\
j \neq i, s \neq c\end{array}$ & $\begin{array}{l}\mu_{i c} \frac{k_{i c}(t)+1}{\sum_{c^{*}=1}^{r} k_{i c}{ }^{*}(t)+1} p_{i c j s}^{-} u\left(k_{i c}(t)\right) \times \\
\quad \times u\left(k_{j s}(t)\right) \Delta t+o(\Delta t)\end{array}$ & $\begin{array}{l}-r_{i c j s}\left(k-I_{i c}-I_{j s}, t\right)+ \\
\quad+v_{i}\left(k-I_{i c}-I_{j s}, t\right)\end{array}$ \\
\hline $\begin{array}{c}(k, t) \rightarrow \\
\left(k-I_{m l}-I_{d h}, t+\Delta t\right) \\
m, d \neq i, l, h \neq c\end{array}$ & $\begin{array}{l}\mu_{m l} \frac{k_{m l}(t)+1}{\sum_{l^{*}=1}^{r} k_{m l^{*}}(t)+1} p_{m l d h}^{-} u\left(k_{m l}(t)\right) \times \\
\quad \times u\left(k_{d h}(t)\right) \Delta t+o(\Delta t)\end{array}$ & $\begin{array}{c}r_{i}(k) \Delta t+ \\
+v_{i}\left(k-I_{m l}-I_{d h}, t\right)\end{array}$ \\
\hline
\end{tabular}

Using the formula for the total probability for the expected value, we get a system of difference-differential equations (DDE) for the expected volume of $v_{i}(k, t)$ in the system $S_{i}$ :

$$
\begin{aligned}
& \frac{d v_{i}(\vec{k}, t)}{d t}=r_{i}(\vec{k})-\left[\lambda^{+}+\lambda^{-}+\sum_{j=1}^{n} \sum_{s=1}^{r} \mu_{j s} u\left(k_{j s}(t)\right)\right] v_{i}(\vec{k}, t)+\sum_{j=1}^{n} \sum_{s=1}^{r} \lambda_{0 j s}^{+} u\left(k_{j s}(t)\right) v_{i}\left(\vec{k}-I_{j s}, t\right)+ \\
& \quad+\sum_{j=1}^{n} \sum_{s=1}^{r}\left[\lambda_{0 j s}^{-}+\mu_{j s} \frac{k_{j s}(t)+1}{\sum_{s^{*}=1}^{r} k_{j s}^{*}(t)+1}\left(p_{j s 0}+\sum_{\substack{m=1 \\
m \neq i=1 \\
m \neq i}}^{n} \sum_{\substack{l=c \\
\text { s. }}}^{r} p_{j s m l}^{-}\left(1-u\left(k_{m l}(t)\right)\right)\right] v_{i}\left(\vec{k}+I_{j s}, t\right)+\right.
\end{aligned}
$$




$$
\begin{aligned}
& +\sum_{\substack{m, d=1 \\
m, d \neq i, m \neq d}}^{n}\left[\mu_{m l} \frac{k_{m l}(t)+1}{\sum_{l^{*}=1}^{r} k_{m l^{*}}(t)+1}\left(p_{m l d h}^{+} v_{i}\left(\vec{k}+I_{m l}-I_{d h}, t\right)+p_{m l d h}^{-} v_{i}\left(\vec{k}+I_{k l}+I_{d h}, t\right)\right)\right]+ \\
& +\sum_{c=1}^{r}\left\{\lambda^{+} p_{0 i c}^{+} u\left(k_{i c}(t)\right) v_{i}\left(\vec{k}-I_{i c}, t\right)+\right. \\
& +\left[\lambda^{-} p_{0 i c}^{-}+\mu_{i c} \frac{k_{i c}(t)+1}{\sum_{\substack{* \\
c^{*}=1}}^{r} k_{i c}(t)+1}\left(p_{i c 0}+\sum_{\substack{m=1 \\
m \neq i}}^{n} \sum_{\substack{l \neq c \\
l \neq c}}^{r} p_{i c m l}^{-}\left(1-u\left(k_{m l}(t)\right)\right)\right] v_{i}\left(\vec{k}+I_{i c}, t\right)+\right. \\
& +\sum_{\substack{j=1 \\
j \neq i}}^{n} \sum_{\substack{s \neq c \\
s \neq c}}^{r}\left[\mu_{j s} \frac{k_{j s}(t)+1}{\sum_{\substack{* \\
s^{*}=1}}^{r} k_{j s}(t)+1} p_{j s i c}^{+} u\left(k_{i c}(t)\right) v_{i}\left(\vec{k}+I_{j s}-I_{i c}, t\right)+\right. \\
& +\mu_{i c} \frac{k_{i c}(t)+1}{\sum_{c^{*}=1}^{r} k_{i c^{*}}(t)+1} p_{i c j s}^{+} u\left(k_{j s}(t)\right) v_{i}\left(\vec{k}-I_{j s}+I_{i c}, t\right)+ \\
& \left.+\mu_{i c} \frac{k_{i c}(t)+1}{\sum_{c^{*}=1}^{r} k_{i c}(t)+1} p_{i c j s}^{-} v_{i}\left(\vec{k}+I_{i c}+I_{j s}, t\right)\right]+\lambda^{+} p_{0 i c}^{+} u\left(k_{i c}(t)\right) r_{0 i c}\left(\vec{k}-I_{i c}, t\right)- \\
& -\left[\mu_{i c} \frac{k_{i c}(t)+1}{\sum_{c^{*}=1}^{r} k_{i c^{*}}(t)+1}\left(p_{i c 0}+\lambda^{-} p_{0 i c}^{-}+\sum_{\substack{m=1 \\
m \neq i}}^{n} \sum_{\substack{l=1 \\
l \neq c}}^{r} p_{i c m l}^{-}\left(1-u\left(k_{m l}(t)\right)\right)\right] R_{i c 0}\left(\vec{k}-I_{i c}, t\right)+\right. \\
& +\sum_{\substack{j=1 \\
j \neq i}}^{n} \sum_{c=1}^{r}\left[\mu_{j s} \frac{k_{j s}(t)+1}{\sum_{\substack{* \\
s^{*}=1}}^{r} k_{j s^{*}}(t)+1} p_{j s i c}^{+} u\left(k_{j s}(t)\right) r_{i c j s}\left(\vec{k}-I_{i c}+I_{j s}, t\right)-\right. \\
& \left.\left.-\mu_{i c} \frac{k_{i c}(t)+1}{\sum_{c^{*}=1}^{r} k_{i c}{ }^{*}(t)+1}\left(p_{i c j s}^{-} r_{i c j s}\left(\vec{k}+I_{i c}+I_{j s}, t\right)+p_{i c j s}^{+} u\left(k_{j s}(t)\right) r_{i c j s}\left(\vec{k}+I_{i c}-I_{j s}, t\right)\right)\right]\right\} \text {. }
\end{aligned}
$$


The number of equations in this system is equal to the number of network states, i.e. for an open network, equal to infinity $\infty$. Formally, the system of equations (1) can be reduced to a system of a countable number of nonlinear inhomogeneous DDE, and solved using numerical methods. But in practice this method can be used only in special cases, when the matrix of coefficients in the system of DDE has a special form.

\section{Analysis of the expected volumes of requests in the systems in the case when their changes from transitions between network states are random variables with given average values}

Let random variable (RV) $\xi_{i c}$ - time of servicing the $c$ type request in the system $S_{i}$, which has the exponential distribution with the cumulative distribution function $F_{\xi_{i c}}(t)=1-e^{-\mu_{i c} t}, i=\overline{1, n}, c=\overline{1, r}$. Consider the dynamics of changes to the overall volume of the requests in the system $S_{i}$ of the network. Let, at the initial moment of time, the volume of requests for this QS be equal to $v_{i 0}$. We will be interested in the volume $V_{i}^{(c)}(\vec{k}, t)$ of $c$-type requests in the $S_{i}$ system at time $t$, when the network was initially in the state of $\vec{k}$ and is associated with transits of $c$-type requests between states of network systems. Changes in the volume of requests in the time interval $[t, t+\Delta t)$ can be described by the formula

$$
V_{i}^{(c)}(\vec{k}, t+\Delta t)=V_{i}^{(c)}(\vec{k}, t)+\Delta V_{i}^{(c)}(\vec{k}, t, \Delta t)
$$

where $\Delta V_{i}^{(c)}(\vec{k}, t, \Delta t)$ - change of $S_{i}$ system volume in the time interval $[t, t+\Delta t)$, related to the transitions between QS network systems of $c$-type requests, $c, i=\overline{1, n}$, $c=\overline{1, r}$. In order to find the volume of $c$-type declarations in the system $S_{i}$, we will save the conditional probabilities of events that may occur at time $\Delta t$ and change the volume of requests for this QS at that time. The following cases are possible:

1) with the probability $\lambda_{0 i c}^{+} \Delta t+o(\Delta t)$ in the system $S_{i}$ enters a positive $c$-type request, which will increase the volume of requests by the size $r_{0 i c}$, where $r_{0 i c}$ a random variable (RV) with the expected value (e.v.) $E\left\{r_{0 i c}\right\}=a_{0 i c}, i=\overline{1, n}$, $c=\overline{1, r}$

2) with the probability $\lambda_{0 i c}^{-} \Delta t+o(\Delta t)$ in the system $S_{i}$ enters a negative $c$-type request, which will reduce the volume of requests by the size $-\bar{r}_{0 i c}$, where $\bar{r}_{0 i c}$ a RV with the e.v. $E\left\{\bar{r}_{0 i c}\right\}=\bar{a}_{0 i c}, i=\overline{1, n}, c=\overline{1, r}$.

Let $\rho_{i c}$ - the probability that on servicing in $S_{i}$ QS there are entries of $c$-type, $i=\overline{1, n}, c=\overline{1, n}$. Let's assume that requests for different streams are expected to be serviced in different queues; all queues are $r$. If the service line becomes free, 
then servicing with the probability $\frac{\rho_{i c}}{\sum_{c=1}^{r} \rho_{i c}}$ will enter the request from queue $c$, $c=\overline{1, r}, i=\overline{1, n}$. 3) with probability $\mu_{i c} \frac{\rho_{i c}}{\sum_{c^{*}=1}^{r} \rho_{i c^{*}}} p_{i c 0} u\left(k_{i c}(t)\right) \Delta t+o(\Delta t)$ the $c$-type request from the

$S_{i}$ system will go to the external environment, the total volume of requests in the system $S_{i}$ will decrease by the size $R_{i c 0}$, where $R_{i c 0}$ - a RV with the e.v. $E\left\{R_{i c 0}\right\}=b_{i c 0}, i=\overline{1, n}, c=\overline{1, r}$;

4) a positive $c$-type request from the $S_{i}$ system will go into the $S_{j}$ system as a $s$-type request with the probability $\mu_{i c} \frac{\rho_{i c}}{\sum_{c^{*}=1}^{r} \rho_{i c^{*}}} p_{i c j s}^{+} u\left(k_{i c}(t)\right) \Delta t+o(\Delta t), i, j=\overline{1, n}$, $s, c=\overline{1, r}, i \neq j, s \neq c$; while the volume of requests in the $S_{i}$ system will decrease by the amount of $R_{i c j s}\left(\xi_{i}\right)$ :

$$
E\left\{R_{i c j s}\left(\xi_{i}\right)\right\}=\int_{0}^{\infty} R_{i c j s}(t) d F_{\xi_{i c}}(t)=\mu_{i c} \int_{0}^{\infty} R_{i c j s}(t) e^{-\mu_{i c} t} d t=a_{i c j s}, i, j=\overline{1, n}, i \neq j
$$

5) with the probability $\mu_{j s} \frac{\rho_{j s}}{\sum_{s^{*}=1}^{r} \rho_{j s^{*}}} p_{j s i c}^{+} u\left(k_{i c}(t)\right) \Delta t+o(\Delta t)$, the negative $s$-type request will pass from the system $S_{j}$ to the system $S_{i}$ as a $c$-type request, while the volume of requests in the $S_{i}$ system will increase by the amount $R_{j s i c}\left(\xi_{j s}\right)$, and the volume of requests in the system $S_{j}$ will decrease by this amount, $E\left\{R_{j s i c}\left(\xi_{j s}\right)\right\}=a_{j s i c}, j=\overline{1, n}, j \neq i ;$

6) a positive $c$-type request from the $S_{i}$ system will pass into the $S_{j}$ system with the probability $\mu_{i c} \frac{\rho_{i c}}{r} p_{i c j s}^{-} \Delta t+o(\Delta t)$ as a negative $s$-type request $i, j=\overline{1, n}$, $\sum_{c^{*}=1}^{r} \rho_{i c}^{*}$

$i \neq j$; the volume of requests in the $S_{i}$ system will decrease by the amount of $\bar{R}_{i c j s}$, where $\bar{R}_{i c j s}-\mathrm{RV}$ with the e.v. $E\left\{\bar{R}_{i c j s}\right\}=c_{i c j s}, i, j=\overline{1, n}, i \neq j, s, c=\overline{1, r}$;

7) with the probability $1-\left[\lambda^{+}+\lambda^{-}+\sum_{i=1}^{n} \sum_{c=1}^{r} \mu_{i c} u\left(k_{i c}(t)\right)\right] \Delta t+o(\Delta t)$, in time $\Delta t$, the network will not change; 
From the above follows

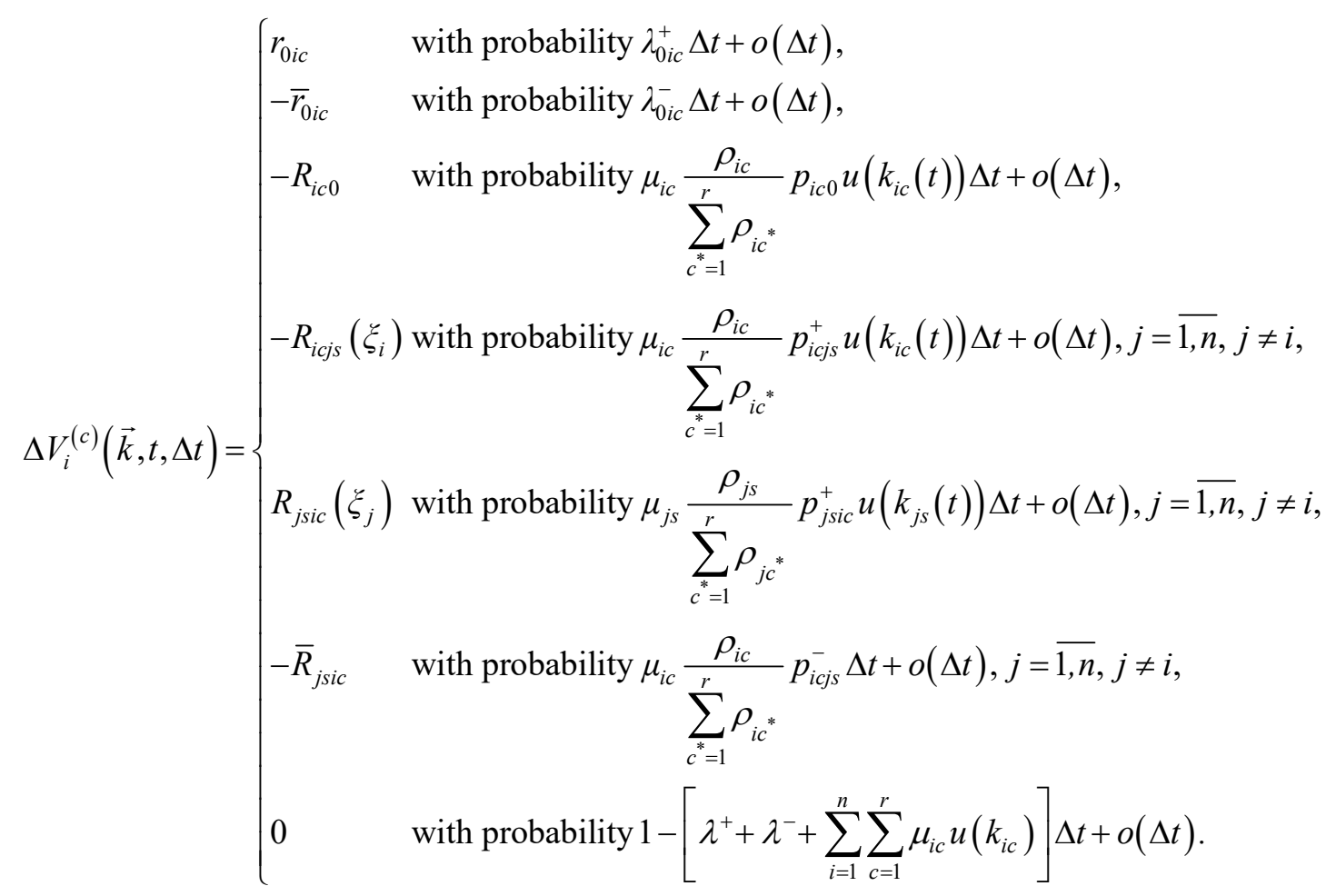

We find the expression for changing the average volume of requests in the system $S_{i}$ at the time $t$. Using (3) for the conditional expected value, we can write:

$$
\begin{gathered}
E\left\{\Delta V_{i}^{(c)}(\vec{k}, t, \Delta t) / \vec{k}(t)\right\}=a_{0 i c}\left(\lambda_{0 i c}^{+} \Delta t+o(\Delta t)\right)-\bar{a}_{0 i c}\left(\lambda_{0 i c}^{-} \Delta t+o(\Delta t)\right)+ \\
-b_{i c 0}\left(\mu_{i c} \frac{\rho_{i c}}{\sum_{\substack{* \\
c^{*}=1}}^{r} \rho_{i c}{ }^{*}} E u\left(k_{i c}(t)\right) p_{i c 0} \Delta t+o(\Delta t)\right)- \\
\sum_{\substack{j=1 \\
j \neq i}}^{n} \sum_{c, s=1}^{r}\left[a_{i c j s}\left(\mu_{i s} \frac{\rho_{i c}}{\sum_{c^{*}=1}^{r} \rho_{i c}} E u\left(k_{i c}^{*}(t)\right) p_{i c j s}^{+} \Delta t+o(\Delta t)\right]+\right.
\end{gathered}
$$




$$
\begin{gathered}
+\sum_{\substack{j=1 \\
j \neq i}}^{n} \sum_{c, s=1}^{r}\left[a _ { j s i c } \left[\mu_{j s} \frac{\rho_{j s}}{\left.\sum_{\substack{*=1 \\
s^{*}=1}}^{r} \rho_{j s^{*}}{ }_{j s i c}^{+} E u\left(k_{j s}(t)\right) \Delta t+o(\Delta t)\right]-}\right.\right. \\
\sum_{\substack{j=1 \\
j \neq i}}^{n} \sum_{c, s=1}^{r}\left[c_{i c j s}\left(\mu_{i c} \frac{\rho_{i c}}{\sum_{\substack{* \\
c^{*}=1}}^{r} \rho_{i c^{*}}} p_{i c j s}^{-} \Delta t+o(\Delta t)\right]\right]
\end{gathered}
$$

By calculating the average value on both sides in (2) with respect to $\vec{k}(t)$, we have:

$$
\begin{aligned}
& v_{i}^{(c)}(\vec{k}, t+\Delta t)=E\left\{V_{i}^{(c)}(\vec{k}, t+\Delta t)\right\}=v_{i}^{(c)}(\vec{k}, t)+\sum_{\vec{k}} P(\vec{k}(t)=\vec{k}) E\left\{\Delta V_{i}^{(c)}(\vec{k}, t, \Delta t)\right\}= \\
& =v_{i}^{(c)}(\vec{k}, t)+\sum_{k_{1}=0}^{\infty} \sum_{k_{2}=0}^{\infty} \ldots \sum_{k_{n}=0}^{\infty} P\left(\vec{k}(t)=\left(k_{1}(t), k_{2}(t), \ldots, k_{n}(t)\right)\right) E\left\{\Delta V_{i}^{(c)}(\vec{k}, t, \Delta t) / \vec{k}(t)=\left(k_{1}(t), k_{2}(t), \ldots, k_{n}(t)\right)\right\}= \\
& =v_{i}^{(c)}(\vec{k}, t)+\left[a_{0 i c} \lambda_{0 i c}^{+}-\bar{a}_{0 i c} \lambda_{0 i c}^{-}-b_{i c 0} \mu_{i c} \frac{\rho_{i c}}{\sum_{c^{*}=1}^{r} \rho_{i c^{*}}} p_{i c 0} E u\left(k_{i c}(t)\right)\right] \Delta t+ \\
& +\sum_{j=1}^{n} \sum_{c, s=1}^{r}\left[-a_{i c j s} \mu_{i c} p_{i c j s}^{+} \frac{\rho_{i c}}{\sum_{j \neq i}^{r} \rho_{i c}} E u\left(k_{j s}(t)\right)+c_{i c j s} \mu_{i c} \frac{\rho_{i c}}{\sum_{c^{*}=1}^{r} \rho_{i c^{*}}} p_{i c j s}^{-}+a_{j s i c} \frac{\rho_{j s}}{\sum_{c^{*}=1}^{r} \rho_{j s s^{*}}} \mu_{j s} p_{j s i c}^{+} E u\left(k_{i c}(t)\right)\right] \Delta t+o(\Delta t), i=\overline{1, n,}
\end{aligned}
$$

i.e.

$$
\begin{aligned}
v_{i}(\vec{k}, t)=\sum_{c=1}^{r} v_{i}^{(c)}(\vec{k}, t)= & v_{i 0}+\sum_{c=1}^{r}\left(a_{0 i c} \lambda_{0 i c}^{+}-\bar{a}_{0 i c} \lambda_{0 i c}^{-}\right) t+\sum_{\substack{j=1 \\
j \neq i}}^{n} \sum_{c, s=1}^{r} c_{i c j s} \mu_{i c} p_{i c j s}^{-} \frac{\rho_{i c}}{\sum_{c=1}^{r} \rho_{i c}} t- \\
& -\sum_{c=1}^{r} b_{i c 0} \mu_{i c} p_{i c 0} \frac{\rho_{i c}}{\sum_{\substack{* \\
c^{*}=1}}^{r} \rho_{i c^{*}}} E u\left(k_{i c}(t)\right)+
\end{aligned}
$$




$$
+\sum_{\substack{j=1 \\ j \neq i}}^{n} \sum_{c, s=1}^{r}\left[-a_{i c j s} \mu_{i c} p_{i c j s}^{+} \frac{\rho_{i c}}{\sum_{\substack{* \\ c^{*}=1}}^{r} \rho_{i c^{*}}} E u\left(k_{i c}(t)\right)+a_{j s i c} \mu_{j s} p_{j s i c}^{+} \frac{\rho_{j s}}{\sum_{\substack{* \\ s^{*}=1}}^{r} \rho_{j s^{*}}} E u\left(k_{j s}(t)\right)\right] .
$$

In the case when it operates under high load conditions, i.e. $\forall t \geq 0, k_{i c}(t)>0$, the last value takes the form:

$$
\begin{aligned}
& v_{i}(\vec{k}, t)=\sum_{c=1}^{r} v_{i}^{(c)}(\vec{k}, t)=v_{i 0}+\sum_{c=1}^{r}\left(a_{0 i c} \lambda_{0 i c}^{+}-\bar{a}_{0 i c} \lambda_{0 i c}^{-}\right) t-\sum_{c=1}^{r} b_{i c 0} \mu_{i c} p_{i c 0} \frac{\rho_{i c}}{\sum_{c^{*}=1}^{r} \rho_{i c^{*}}} t+ \\
& +\sum_{\substack{j=1 \\
j \neq i}}^{n} \sum_{c, s=1}^{r}\left[\left(c_{i c j s} p_{i c j s}^{-}-a_{i c j s} p_{i c j s}^{+}\right) \mu_{i c} \frac{\rho_{i c}}{\sum_{\substack{* \\
c^{*}=1}}^{r} \rho_{i c^{*}}}+a_{j s i c} \mu_{j s} p_{j s i c}^{+} \frac{\rho_{j s}}{\sum_{\substack{* \\
s^{*}=1}}^{r} \rho_{j s^{*}}}\right] t .
\end{aligned}
$$

\section{Conclusions}

Further research in this direction may be related to the analysis of any (non-Markovian) networks with random volume requests and Markovian networks with other different features.

\section{References}

[1] Tikhonenko, O. (2006). Metody probalistyczne analizy systemów informacyjnych. Warszawa: Akademicka Oficyna Wydawnicza EXIT.

[2] Matalytski, M., \& Naumenko, V. (2016). Stochastic Networks with Nonstandard Moving Customers. Grodno: GrSu (In Russian).

[3] Matalytski, M., \& Naumenko, V. (2015). Zastosowanie HM-sieci kolejkowych dla wyznaczenia objętości pamięci systemów informacyjnych. Studia Informatica, 35(3), 63-69.

[4] Matalytski, M., \& Zajac, P. (2017). Finding of expected volumes of request in systems of the queueing network with limited amount of spaces of expectations. Studia Informatica, 38(1B), 89-104.

[5] Matalytski, M., \& Zajac, P. (2017). Application of HM-networks with impatient claims in finding the memory capacity in the information systems. Journal of Applied Mathematics and Computational Mechanics, 16(3), 47-59.

[6] Matalytski, M., Zajac, P., \& Kopats, D. (2018). Application of HM-networks with unreliable systems for finding the memory capacity in the information systems. Journal of Applied Mathematics and Computational Mechanics, 17(2), 51-63. 
[7] Gelenbe, E. (1991). Product form queueing networks with negative and positive customers. Journal of Applied Probability, 28, 656-663.

[8] Fourneau, J.M. (1996). G-networks with multiple classes of negative and positive customers. Theoretical Computer Science, 155(1), 141-156.

[9] Gelenbe, E. (2002). G-Networks: Multiple Classes of Positive Customers, Signals, and Product Form. Results Performance Evaluation of Complex Systems: Techniques and Tools. Lecture Notes in Computer Science, 1-16. 